\title{
Erastin triggers autophagic death of breast cancer cells by increasing intracellular iron levels
}

\author{
MENGXIN LI ${ }^{1,2}$, XUANZHONG WANG ${ }^{2,3}$, SHAN LU $^{2,3}$, CHUAN HE $^{2,3}$, CHONGCHENG WANG $^{2,3}$, \\ LEI WANG ${ }^{2,3}$, XINYU WANG ${ }^{4}$, PENGFEI GE ${ }^{2,3}$ and DONG SONG ${ }^{1}$ \\ ${ }^{1}$ Department of Breast Surgery; ${ }^{2}$ Research Center of Neuroscience; ${ }^{3}$ Department of Neurosurgery, \\ First Hospital of Jilin University, Changchun, Jilin 130021; ${ }^{4}$ Department of Radiotherapy, \\ The Second Hospital of Jilin University, Changchun, Jilin 130022, P.R. China
}

Received February 1, 2020; Accepted June 19, 2020

DOI: $10.3892 / 01.2020 .11918$

\begin{abstract}
Erastin is a small molecular compound that induces ferroptosis by binding to voltage-dependent anion-selective channel protein (VDAC)2, VDAC3 and solute carrier family 7 member 5 inhibiting the cystine/glutamate antiporter. However, to the best of our knowledge, the mechanism of erastin-induced breast cancer cell death remains unclear. In present study aimed to explore the underlying mechanisms of the antitumor effects of erastin on breast cancer cells. Cellular viability was assessed using an MTT assay, a lactate dehydrogenase cytotoxicity assay kit was used to determine the cell death rate, the intracellular $\mathrm{Fe}^{2+}$ levels were determined using an iron colorimetric assay kit and western blotting was used to estimate the changes of autophagy-associated proteins levels. The present study demonstrated that erastin inhibited the viability of breast cancer cells and induced breast cancer cell death in a dose-dependent manner. Additionally, autophagy was activated by erastin, as demonstrated by upregulated expression levels of autophagy-associated proteins in breast cancer cells. Bafilomycin A1, 3-methyladenine and knockdown of autophagy related (ATG)5 with small interfering RNA prevented erastin-induced breast cancer cell death and inhibited the erastin-induced changes in the expression levels of the autophagy-associated proteins beclin1, ATG5, ATG12, microtubule-associated proteins 1A/1B light chain 3B (LC3B) and P62. Furthermore, erastin-induced breast cancer cell death was inhibited by an iron chelator, deferoxamine, which inhibited the increases of erastin-induced iron levels and inhibited the erastin-induced changes in the expression levels of the autophagy-related proteins beclin1, ATG5, ATG12, LC3B and $\mathrm{P} 62$. In summary, erastin triggered autophagic death in breast cancer cells by increasing intracellular iron levels.
\end{abstract}

Correspondence to: Dr Dong Song, Department of Breast Surgery, First Hospital of Jilin University, 71 Xinmin Avenue, Changchun, Jilin 130021, P.R. China

E-mail: songdong@jlu.edu.cn

Key words: erastin, autophagy, ferroptosis, breast carcinoma

\section{Introduction}

Breast cancer became the primary cause of mortality among women worldwide in 2017 (1). According to the different subtypes of breast carcinoma, there are four key treatments of breast cancer, including surgery, chemotherapy, endocrine therapy and radiotherapy (2). However, prognosis remains poor due to increasing resistance to apoptosis and recurrence (3). Therefore, novel treatments that are less toxic and more sensitive are urgently required.

Erastin is a small molecular compound that inhibits the solute carrier family 7 member 5 inhibiting the cystine/glutamate antiporter (system xc-) and induces ferroptosis by binding to voltage-dependent anion-selective channel protein (VDAC) 2 or VDAC3, causing mitochondrial oxidative injury (4). Erastin also binds to solute carrier family 7 member 5 (SLC7A5), which interferes with cystine uptake via the SLC3BA2/SLC7A11 complex in trans to deplete glutathione (GSH) (5). In contrast to other forms of cell death, ferroptosis is a unique type of programmed cell death that has two major characteristics; lipid peroxide accumulation and iron dependency (6). Previous studies have demonstrated that ferroptosis is often accompanied by autophagy and can be inhibited by autophagy inhibitors (7-9). As an inducer of ferroptosis, erastin has been shown to induce ferroptosis in oncogenic RAS mutation cell lines and in other cancer cells, including liver cancer (10), acute lymphoblastic leukemia (11) and rhabdomyosarcoma (12). Although erastin activates ferroptosis in triple-negative breast cancer cells by suppressing the expression of glutathione peroxidase 4 and upregulating the expression of cysteine dioxygenase (13), understanding is limited regarding the effect of erastin treatment or the mechanism of erastin in other types of breast cancer cells.

Ferroptosis is considered to be a type of reactive oxygen species (ROS)-dependent regulated necrosis that is accompanied by intracellular iron accumulation (14). Iron exists as $\mathrm{Fe}^{2+}$ and $\mathrm{Fe}^{3+}$ in cells. Free $\mathrm{Fe}^{2+}$ catalyzes the formation of hydroxyl radicals and hydroxide from hydrogen peroxide which is termed the Fenton reaction (15). During the catalytic cycle of the Fenton reaction, $\mathrm{Fe}^{3+}$ can be recycled to reproduce $\mathrm{Fe}^{2+}$ via superoxide radicals. Additionally, $\mathrm{Fe}^{2+}$ catalyzes the lipid peroxidation of unsaturated fatty acids (15). When ROS 
levels exceed the antioxidant capacity of cells, it causes oxidative stress, which triggers oxidative damage to proteins, DNA and lipids (15). Previous studies have reported that autophagy accelerates ferroptosis by: i) generating lysosomal ROS (16); ii) accumulating labile iron via nuclear receptor coactivator 4-mediated ferritinophagy (17); iii) promoting lipid peroxidation via Ras-related protein Rab-7-mediated lipophagy (18); iv) promoting GSH depletion via beclin1-mediated system xc-inhibition (19); v) promoting lysosomal cell death via signal transducer and activator of transcription 3-mediated cathepsin $\mathrm{B}$ release (20); and iv) contributing to chaperone-mediated autophagy glutathione peroxidase 4 degradation via heat shock protein 90-mediated lysosome-associated membrane protein 2A stability (21). Although numerous studies have investigated the mechanism of erastin in ferroptosis-associated pathways, to the best of our knowledge, the relationship between intracellular iron levels and erastin-induced autophagy remains unclear in breast cancer cells.

The present study therefore investigated the changes of iron levels in erastin-induced autophagy and aimed to elucidate its underlying mechanism using human MCF-7 and MDA-MB-231 cell lines.

\section{Materials and methods}

Reagents. Erastin, 3-methyladenine (3-MA) and bafilomycin A1 (Baf-A1) were purchased from Selleck Chemicals. Anti-autophagy related (ATG)12 (cat. no. 4180) was purchased from Cell Signaling Technology, Inc. Deferoxamine (DFO), anti-p62 (cat. no. ab56416) and anti-ATG5 (cat. no. ab221604) were purchased from Abcam. Anti-beclin 1 (cat. no. B6061) and anti-microtubule-associated proteins $1 \mathrm{~A} / 1 \mathrm{~B}$ light chain 3B (LC3B) (cat. no. 17543) were obtained from Sigma-Aldrich; Merck KGaA. Anti- $\beta$-actin (cat. no. AF5001), and horseradish peroxidase-conjugated anti-mouse IgG (cat. no. A0216) and anti-rabbit IgG (cat.no. A0208) were purchased from Beyotime Institute of Biotechnology.

Cell lines and culture. Human MCF-7 and MDA-MB-231 cell lines were purchased from the Shanghai Institute of Cell Biology at the Chinese Academy of Sciences. All cells were cultured in DMEM (SH 30243.01; Hyclone; Cyvita) supplemented with $10 \%$ heat-inactivated fetal bovine serum (FB15015; http://zn.clarkbio.com/Clark), 2 mmol/l glutamine, penicillin $(100 \mathrm{U} / \mathrm{ml})$ and streptomycin $(100 \mu \mathrm{g} / \mathrm{ml})$. Cells treated with $5 \mathrm{mmol} / 13-\mathrm{MA}, 1.5 \mu \mathrm{mol} / \mathrm{l}$ Baf-A1 or $500 \mu \mathrm{mol} / 1$ DFO for $1 \mathrm{~h}$ prior to erastin treatment which lasted for $24 \mathrm{~h}$ with different concentrations (MCF-7 cells were treated with 40 and $80 \mu \mathrm{mol} / 1$; MDA-MB-231 cells were treated with 20 and $40 \mu \mathrm{mol} / 1$ ) at $37^{\circ} \mathrm{C}$ and $5 \% \mathrm{CO}_{2}$ in a humid environment and used for experimentation when entering the mid-log phase. A light microscope was used at magnification, $\mathrm{x} 400$ to observe cellular changes.

Cell viability assay. MCF-7 $\left(6 \times 10^{3}\right.$ cells/well) and

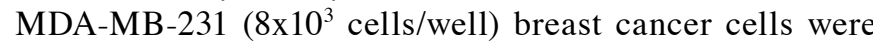
seeded into 96 -well microplates, cultured at $37^{\circ} \mathrm{C}$ for $24 \mathrm{~h}$ and treated with $5 \mathrm{mmol} / 13-\mathrm{MA}, 1.5 \mu \mathrm{mol} / \mathrm{l} \mathrm{Baf}-\mathrm{A} 1$ or $500 \mu \mathrm{mol} / 1$ DFO for $1 \mathrm{~h}$ prior to erastin treatment which lasted for $24 \mathrm{~h}$ with two values separately (MCF-7 cells were treated with
40 and $80 \mu \mathrm{mol} / 1$; MDA-MB-231 cells were treated with 20 and $40 \mu \mathrm{mol} / \mathrm{l}$ ) at $37^{\circ} \mathrm{C}$ and $5 \% \mathrm{CO}_{2}$ in a humid environment. Cellular viability was assessed by performing an MTT assay (DMSO was used to dissolve the purple formazan), the results of which were expressed as a ratio to the absorbance of control cells at $490 \mathrm{~nm}$. Absorbances were measured at $490 \mathrm{~nm}$ using an automatic multi-well spectrophotometer (Bio-Rad Laboratories, Inc.). The $\mathrm{IC}_{50}$ values were calculated using GraphPad Prism 6 software (GraphPad Software, Inc.), and the mean $\mathrm{IC}_{50}$ value of four experiments was presented.

Lactate dehydrogenase $(\mathrm{LDH})$ release cell death assay. The LDH cytotoxicity assay kit (cat. no. C0017; Beyotime Institute of Biotechnology) was used to determine the cell death rate. According to the manufacturer's protocol, the absorbance of each sample was measured at $490 \mathrm{~nm}$ using an automatic multi-well spectrophotometer. The cell death ratio was calculated using the following formula: Cell death ratio $(\%)=$ $\left(\mathrm{A}_{\text {sample }}-\mathrm{A}_{\text {control }} / \mathrm{A}_{\max }-\mathrm{A}_{\text {control }}\right) \times 100 \%$. Where $\mathrm{A}_{\text {sample }}$ is the sample absorbance value, $A_{\text {control }}$ is the absorbance value of the control group and $\mathrm{A}_{\max }$ is the absorbance value of the positive group.

Iron assay. Intracellular $\mathrm{Fe}^{2+}$ levels were determined using an iron colorimetric assay kit (cat. no. K390) purchased from BioVision, Inc. According to the manufacturer's instructions, MCF-7 and MDA-MB-231 breast cancer cells were collected and separated into untreated, erastin-treated and DFO-pretreated groups before the cells were added to iron assay buffer, homogenized on ice and centrifuged at $16,000 \times \mathrm{g}$ for $10 \mathrm{~min}$ at $4^{\circ} \mathrm{C}$ to obtain the supernatant. Samples $(50 \mu \mathrm{l} /$ well) were then incubated with $50 \mu \mathrm{l}$ assay buffer in a 96 -well microplate for $30 \mathrm{~min}$ at $25^{\circ} \mathrm{C}$. Samples were subsequently incubated with $100 \mu \mathrm{l}$ iron probe in the dark for $60 \mathrm{~min}$ at $25^{\circ} \mathrm{C}$ and assessed with a microplate reader at a wavelength of $593 \mathrm{~nm}$. Absorbance values were calibrated to a standard concentration curve to calculate the concentration of iron. The results are expressed as a ratio to the concentration of control cells.

Transfection of small interfering RNA (siRNA). Transfection with ATG5 siRNA $(5 \mu \mathrm{g} / \mu \mathrm{l})$ was performed using Lipofectamine $^{\circledR} 3000$ (Invitrogen; Thermo Fisher Scientific, Inc.), according to the manufacturer's protocol. ATG5 siRNA (5'-GACGUUGGUAACUGACAAATT-3') and scrambled siRNA (5'-UUCUCCGAACGUGUCACGUTT-3') were purchased from Shanghai GenePharma Co., Ltd. MCF-7 and MDA-MB-231 cells were separately seeded into a 6-well plate at $6 \times 10^{4}$ and $8 \times 10^{4}$ cells per well overnight. The following day, cells were transfected with ATG5-specific siRNAs and scrambled siRNAs for $48 \mathrm{~h}$. The efficiency of transfection was determined by western blotting.

Gel electrophoresis and western blotting. MCF-7 and MDA-MB-231 breast cancer cells treated with eastin alone, $5 \mathrm{mmol} / 13-\mathrm{MA}, 1.5 \mu \mathrm{mol} / 1 \mathrm{Baf}-\mathrm{A} 1$, ATG $5 \mathrm{siRNA}$ or $500 \mu \mathrm{mol} / 1$ DFO for $1 \mathrm{~h}$ prior to erastin were collected and homogenized as described previously (22). Homogenates were centrifuged at $1,000 \mathrm{x}$ for $10 \mathrm{~min}$ at $4^{\circ} \mathrm{C}$ to obtain the supernatant, and the protein content of the supernatant was determined using BCA Protein assay kit (Beyotime Institute of Biotechnology). Equal 
quantities of protein including anti-ATG5 (55 kd), anti-ATG12 $(55 \mathrm{kd})$, anti-P62 (62 kd), anti-Beclin1 (69 kd) and anti-LC3B (14 and $16 \mathrm{kd})$ were electrophoresed on 8-12\% sodium dodecyl sulfate-polyacrylamide gels based on the molecular weight of the target protein and transferred to PVDF membranes (EMD Millipore). The membranes were then blocked with $5 \%$ skimmed milk in PBS for $2 \mathrm{~h}$ at room temperature and incubated overnight at $4{ }^{\circ} \mathrm{C}$ with primary antibodies, including anti-ATG5 $(1: 1,000)$, anti-ATG12 $(1: 1,000)$, anti-P62 (1:1,000), anti-Beclin-1 $(1: 1,000)$ and anti-LC3B $(1: 1,000)$. The membranes were washed with PBS-0.1\% Tween-20 (PBS-T) buffer for $30 \mathrm{~min}$ at $25^{\circ} \mathrm{C}$ prior to incubation with horseradish peroxidase-conjugated goat anti-rabbit $\operatorname{IgG}(1: 1,000)$ or anti-mouse $\operatorname{IgG}(1: 1,000)$ at $25^{\circ} \mathrm{C}$ for $2 \mathrm{~h}$. Membranes were subsequently washed with PBS-T buffer and immunoreactive proteins were visualized on a chemi-luminescence developer (Chemiscope 5300; Clinx Science Instruments, Co., Ltd.) with an enhanced chemiluminescence reagent (P10300; NCM Biotech). Densitometry was performed using ImageJ software v1.46 (National Institutes of Health).

Statistical analysis. All data was obtained from at least four independent experiments and are expressed as the mean \pm standard deviation. Statistical analyses were performed with Microsoft Excel 2010 (Microsoft Corporation) and GraphPad Prism 6 software (GraphPad Software, Inc.). Statistical comparisons were made using one-way ANOVA with Tukey's post hoc test. $\mathrm{P}<0.05$ was considered to indicate a statistically significant difference.

\section{Results}

Erastin inhibits viability and induces breast cancer cell death. To investigate the toxic effect of erastin on breast cancer cells, an MTT assay was performed to examine the viability of erastin-treated MCF-7 and MDA-MB-231 cells. Following treatment with $10,20,40$ and $80 \mu \mathrm{mol} / 1$ erastin for $24 \mathrm{~h}$, cell viability was significantly reduced in MCF-7 and MDA-MB-231 cells compared with untreated cells (Fig. 1A). The $\mathrm{IC}_{50}$ values of erastin in MDA-MB-231 and MCF-7 cells at $24 \mathrm{~h}$ were 40 and $80 \mu \mathrm{mol} / 1$, respectively. Therefore, erastin at these concentrations was used for subsequent experiments.

A LDH release assay was performed to examine erastin-induced death in MDA-MB-231 and MCF-7 cells. MDA-MB-231 and MCF-7 cells were treated with erastin at their respective $\mathrm{IC}_{50}$ concentrations for 3, 6, 12 and $24 \mathrm{~h}$. The results at $24 \mathrm{~h}$ demonstrated significant increases in cellular death in each cell line following treatment with 10,20,40 and $80 \mu \mathrm{mol} / 1$ erastin compared with untreated cells (Fig. 1B). These data indicated that erastin inhibited the viability of breast cancer cells and triggered breast cancer cell death

Erastin upregulates the expression of autophagy-associated proteins. To further investigate whether erastin activates autophagy in breast cancer cells, western blotting was performed to analyze the expression of autophagy-associated proteins. Following erastin treatment, the expression of autophagy-associated proteins in breast cancer cells, including beclin-1, ATG5, ATG12 and LC3B, were all significantly increased. Additionally, p62 levels, as a substrate of autophagy, were significantly downregulated in erastin-treated cells (Fig. 1C and D).

Inhibition of autophagy prevents erastin-induced death in breast cancer cells. 3MA and Baf-A1 are often used to inhibit autophagy activation (5). 3MA inhibits autophagy initiation (23), and Baf-A1 disturbs the fusion of autophagosomes with lysosomes (24). The inhibitory effects of 3MA or Baf-A1 on autophagy activation are also decided by their concentrations and treatment times. Higher concentrations of 3MA or Baf-A1 and longer incubation times lead to obvious changes in the baseline level of autophagy-related proteins $(23,24)$. In the present study, the cells were incubated with $5 \mathrm{mmol} / 13 \mathrm{MA}$ and $1.5 \mu \mathrm{mol} / 1 \mathrm{Baf}-\mathrm{A} 1$ for $25 \mathrm{~h}$, which did not exhibit marked inhibition on the baseline levels of autophagy-related proteins (Fig. 2A-D). To further elucidate erastin activated autophagy, cells were treated with $5 \mathrm{mmol} / \mathrm{l} 3-\mathrm{MA}$ for $1 \mathrm{~h}$ prior to erastin treatment. Cells were additionally pre-treated with $1.5 \mu \mathrm{mol} / 1$ Baf-A1, an inhibitor of autophagy, for $1 \mathrm{~h}$ prior to erastin treatment. The results of the $\mathrm{LDH}$ release assay revealed that cells pre-treated with 3-MA or Baf-A1 were significantly more resistant to erastin-induced death compared with cells only treated with erastin (Fig. 2E).

Inhibition of autophagy inhibits the erastin-induced expressional upregulation of autophagy-associated proteins. As aforementioned, the results of the current study demonstrated that autophagy-associated proteins (beclin1, ATG5, ATG12, LC3B and P62) were affected by erastin. However, 3-MA treatment prevented cell death and inhibited the upregulation of autophagy-associated proteins induced by erastin. Western blotting revealed that the expression levels of beclin1, ATG5, ATG12 and LC3B were significantly downregulated, while P62 was significantly upregulated when cells were pre-treated with 3-MA compared with those only treated with erastin (Fig. 2A and B). Baf-A1 treatment demonstrated similar effects to that of 3-MA at a concentration of $1.5 \mu \mathrm{mol} / 1$ on the expression of P62. However, in contrast to 3-MA, the main effect of Baf-A1 is to inhibit the fusion of autophagosome and autolysosome (24), which resulted in a significant increase of LC3B in cells pre-treated with Baf-A1 compared with cells only treated with erastin (Fig. 2C and D). Therefore, it was unnecessary in the present study to detect the expression of ATG5, ATG12 and Beclin-1.

Knockdown of ATG5 with siRNA prevents erastin-induced breast cancer cell death. To further investigate erastin induced autophagic death in breast cancer cells, ATG5 was knocked-down with siRNA. The results demonstrated that ATG5-knockdown significantly decreased the cell death ratio of erastin-treated cells (Fig. 2F) and significantly inhibited the erastin-induced changes of autophagy-related proteins (Fig. 2G and H).

Mitigation of erastin-induced iron level increases inhibits erastin-induced autophagy. To investigate whether the levels of intracellular iron are intrinsically associated with erastin-induced autophagy, an iron assay was performed to assess intracellular iron accumulation after treatment with erastin. The results revealed that when compared with the 

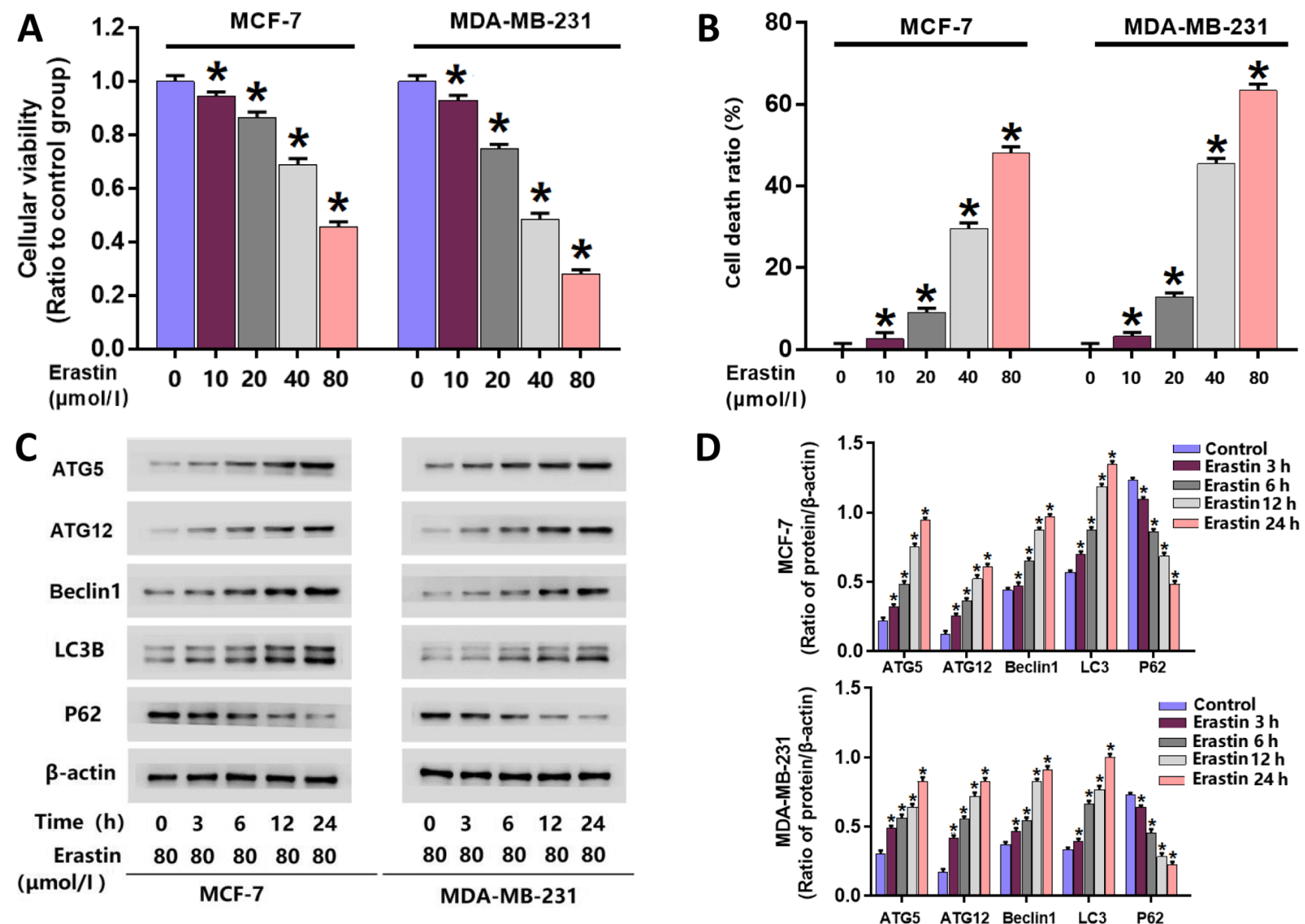

Figure 1. Erastin inhibits viability, induces death and activates autophagy in breast cancer cells. (A) MTT assay demonstrated that erastin inhibited the viability of MCF-7 and MDA-MB-231 breast cancer cells in a dose-dependent manner. (B) LDH release assay demonstrated that erastin enhanced breast cancer cell death in a dose-dependent manner. (C) Western blotting and (D) quantification demonstrated that erastin upregulated the expression levels of beclin1, ATG5, ATG12 and LC3B, and downregulated the expression of P62 in a time-dependent manner. "P<0.01 vs. control untreated group. ATG, autophagy related; LC3B, microtubule-associated proteins $1 \mathrm{~A} / 1 \mathrm{~B}$ light chain $3 \mathrm{~B}$.

control group, iron levels significantly increased in cells incubated with erastin for $24 \mathrm{~h}$. Furthermore, when the concentration of erastin was increased from 40 to $80 \mu \mathrm{mol} / 1$, iron levels significantly increased further. The data indicated that erastin-induced an increase in intracellular irons in each breast cancer cell line in a concentration- and incubation time-dependent manner (Fig. 3A).

To elucidate the importance of iron accumulation, cells were treated with iron chelator DFO at $500 \mu \mathrm{mol} / 1$ for $1 \mathrm{~h}$ and subsequently incubated with erastin for $24 \mathrm{~h}$. Similarly with 3MA and Baf-A1, the dose of DFO used in the current study was $500 \mu \mathrm{mol} / 1$, which did not show any inhibitory effect on cellular viability or the baseline level of autophagy-related proteins (Fig. 3B-E). The results demonstrated that DFO significantly inhibited the erastin-induced increase of intracellular $\mathrm{Fe}^{2+}$ iron in MDA-MB-231 and MCF-7 cells (Fig. 3D). Additionally, the LDH release assay demonstrated that erastin-induced breast cancer cell death was significantly inhibited in the presence of DFO (Fig. 3B). Consistently, observation under a light microscope demonstrated that DFO pre-treatment markedly reversed the erastin-induced cellular changes (Fig. 3F).

To determine the relationship between autophagy and erastin-induced increases of intracellular iron, western blotting was performed to assess the changes of autophagy-associated proteins in each breast cancer cell line following pre-treatment with DFO. The results demonstrated that DFO significantly inhibited the erastin-induced changes in the autophagy-associated protein expression levels (Fig. 3C and E). These data suggested that DFO treatment inhibited erastin-induced autophagy by mitigating erastin-induced iron levels.

\section{Discussion}

The results of the current study demonstrated that erastin inhibited the viability of breast cancer cells, triggered breast cancer cell death and increased intracellular iron levels in a time or dose-dependent manner. In erastin-treated cells, the protein expression of autophagy-associated beclin1, ATG5, ATG12 and LC3B was increased, while levels of P62 were decreased. However, these effects on LC3B and P62 could be inhibited by 3-MA pretreatment and ATG5-knockdown via siRNA. The mitigation of erastin-induced iron levels via DFO pre-treatment inhibited erastin-induced autophagy-associated protein alterations and mitigated cell death. Taken together, the results indicated that erastin induces autophagic breast cancer cell death by improving intracellular iron levels. 

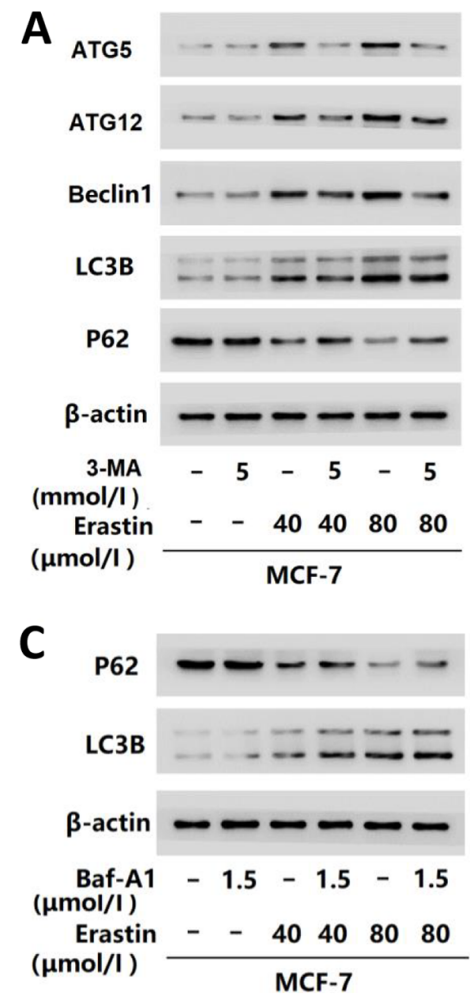

\section{$\mathbf{E}$}

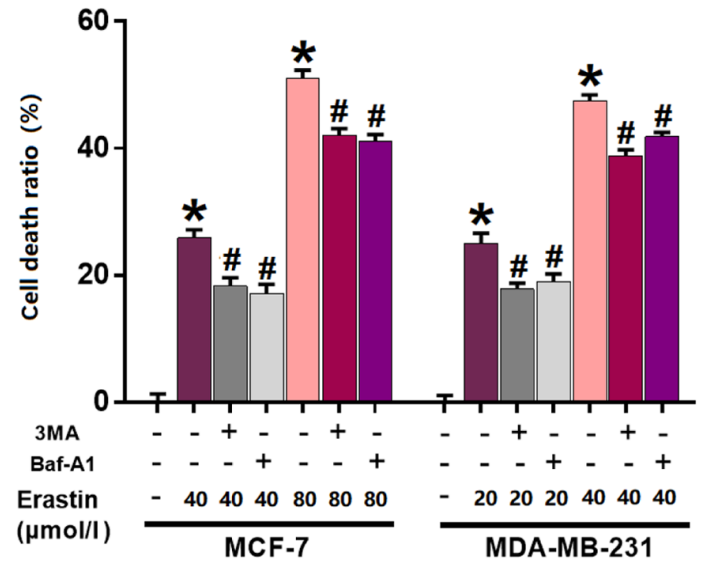

G

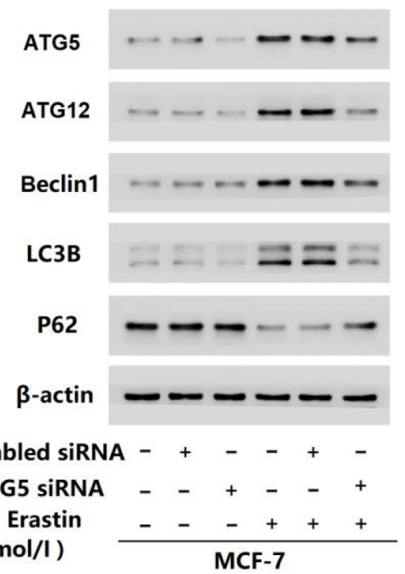

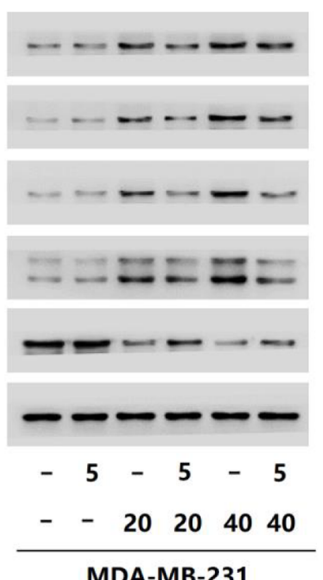

MDA-MB-231
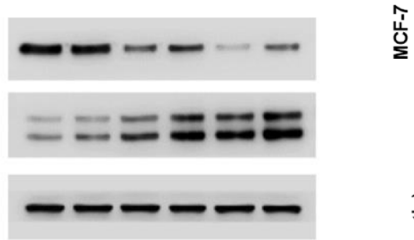

$-1.5-1.5-1.5$

- 20204040

MDA-MB-231

B

D
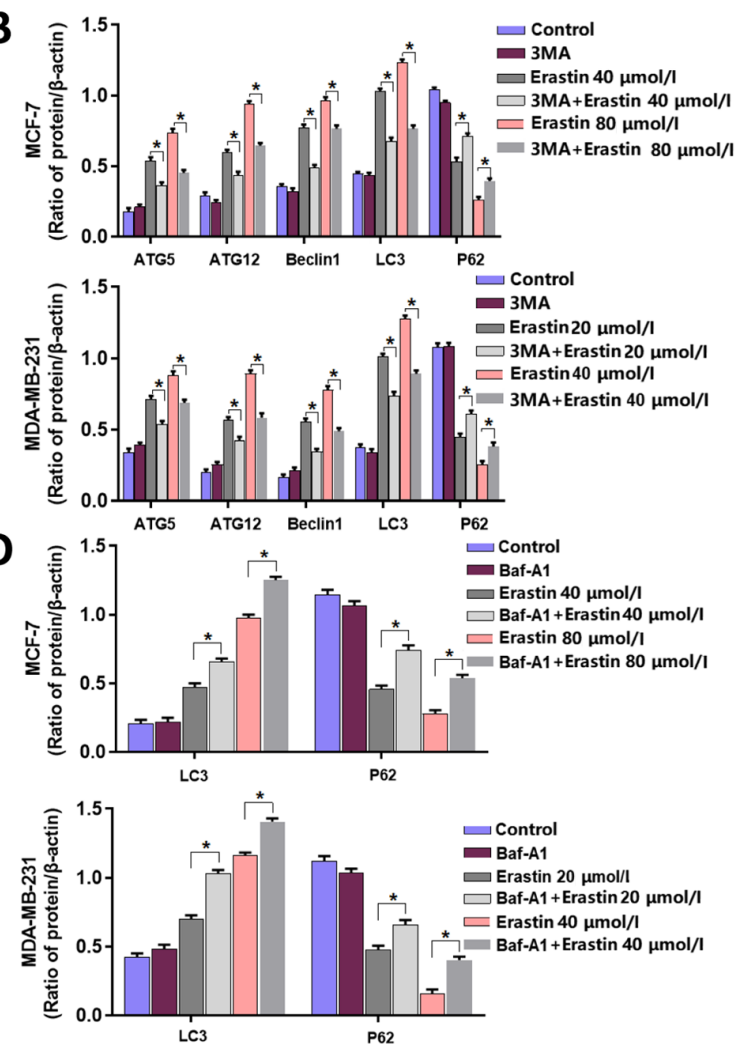

$\mathbf{F}$

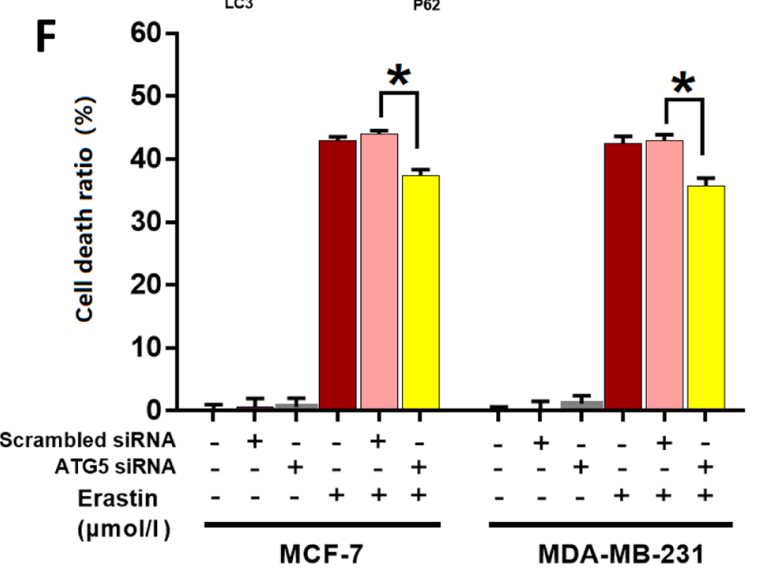

H
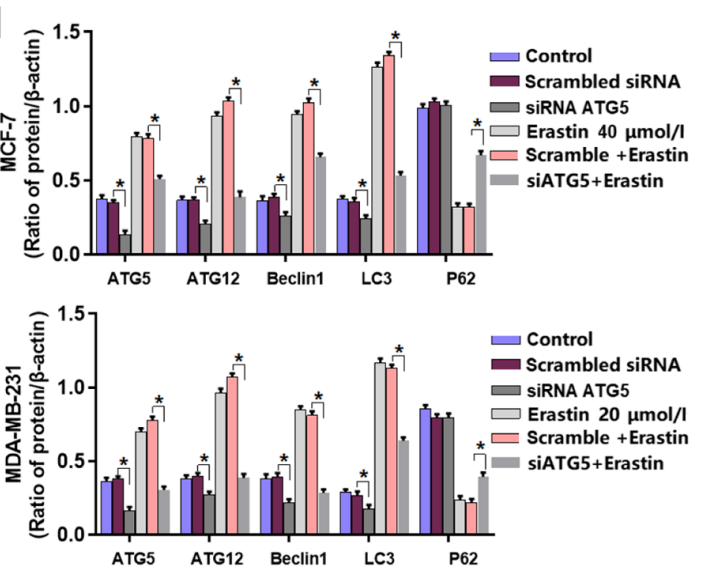

Figure 2. Inhibition of autophagy prevents breast cancer cell death induced by erastin. (A) Western blotting and (B) quantification revealed that the changes in autophagy-related protein expression levels induced by erastin were suppressed by 3-MA. (C) Western blotting and (D) quantification revealed that Baf-A1 inhibited the downregulation of p62 and increased LC3B protein level. (E) LDH release assay demonstrated that erastin-induced cell death was significantly alleviated by pretreatment with $3-\mathrm{MA}$ and Baf-A1. ${ }^{\text {"P }}<0.01$ vs. control untreated group. ${ }^{~} \mathrm{P}<0.01$ vs. erastin only group. (F) LDH release assay demonstrated that knockdown of ATG5 significantly prevented erastin-induced cell death. (G) Western blotting and (H) quantification revealed that the changes in autophagy-related protein expression levels induced by erastin were suppressed by knockdown of ATG5 with siRNA. "P $<0.01$. LDH, lactate dehydrogenase; 3-MA, 3-methyladenine; Baf-A1, bafilomycin A1; ATG, autophagy related; LC3B, microtubule-associated proteins 1A/1B light chain 3B; siRNA, small interfering RNA. 
A

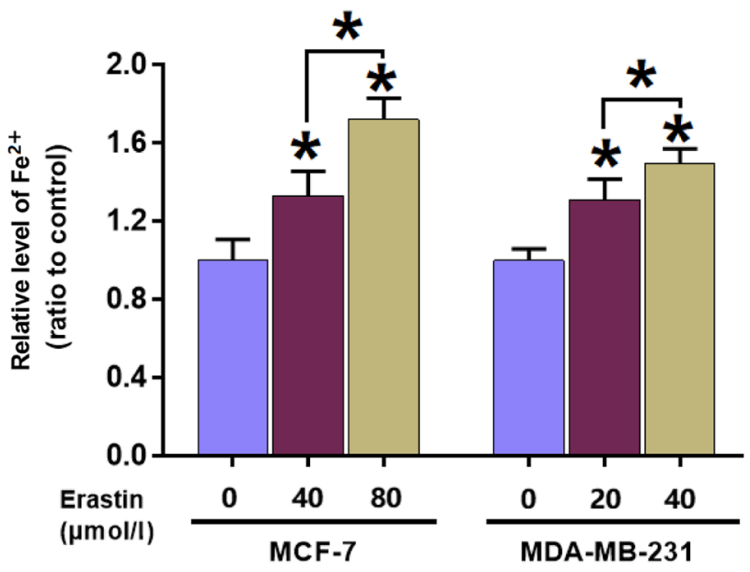

C

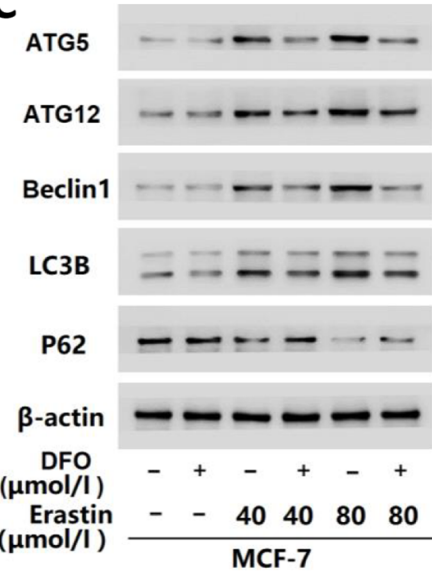

$\mathbf{E}$

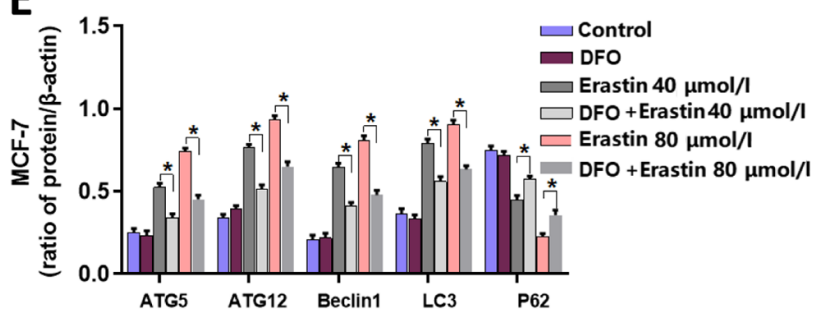

$\mathbf{F}$
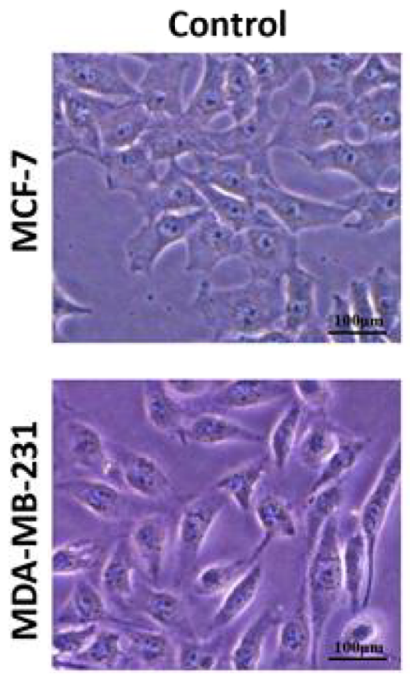

\section{Erastin}
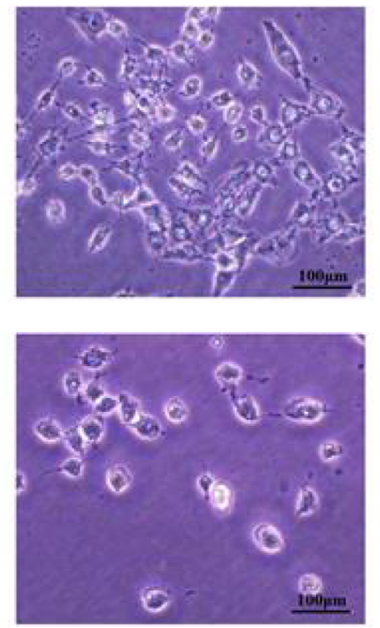

B

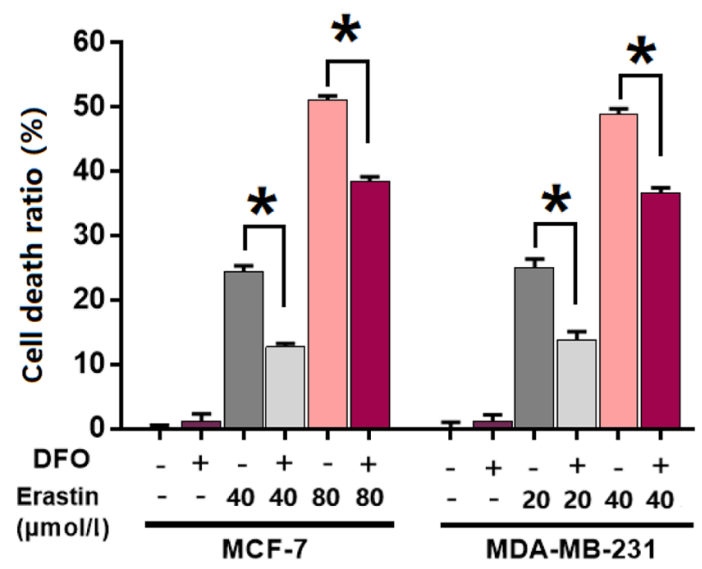

D

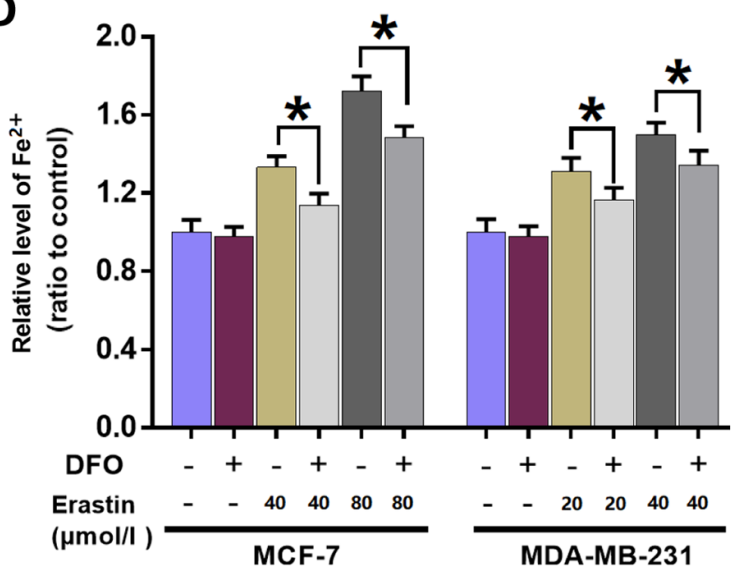

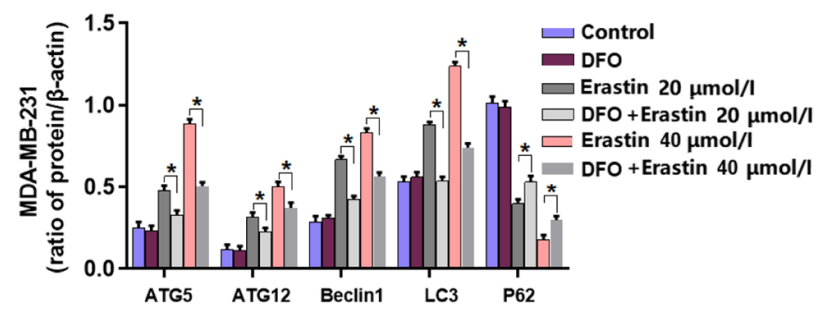
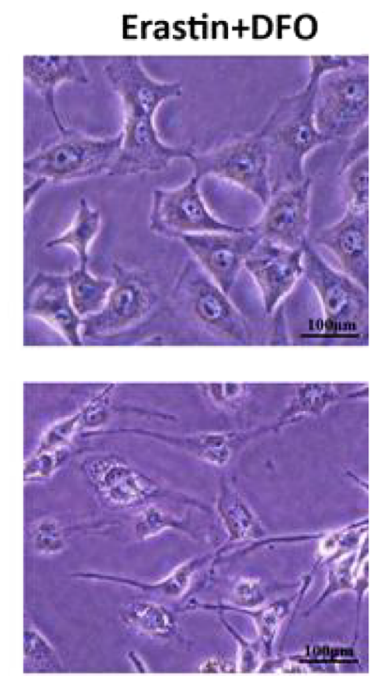

Figure 3. Mitigation of erastin-induced increases in irons levels inhibits erastin-induced autophagy. (A) Erastin increased the levels of intracellular Fe ${ }^{2+}$ in MCF-7 and MDA-MB-231 cells in a dose-dependent manner. (B) LDH release assay demonstrated that the breast cancer cell death induced by erastin was inhibited in the presence of DFO. (C) Western blotting and (E) quantification demonstrated that the upregulation of autophagy-related protein expression levels induced by erastin was suppressed by DFO. (D) Pretreatment with $500 \mu \mathrm{mol} / 1 \mathrm{DFO}$ for $1 \mathrm{~h}$ mitigated the erastin-induced increases of intracellular Fe ${ }^{2+}$ (F) Representative light microscope images of MCF-7 and MDA-MB-231 breast cancer cells. The majority of cells treated with erastin alone became round in shape and smaller in size, which was prevented by pretreatment with DFO. Scale bar, $100 \mu \mathrm{m}$. ${ }^{*} \mathrm{P}<0.01$ vs. untreated control group or as indicated. DFO, deferoxamine; LDH, lactate dehydrogenase; ATG, autophagy related; LC3B, microtubule-associated proteins 1A/1B light chain 3B. 
The relationship between iron and breast cancer is complex. Huang et al (25) hypothesized that iron deficiency contributes to the high recurrence of breast cancer in premenopausal women via increased serum vascular endothelial growth factor concentrations. Additionally, it was predicted that iron load may serve a role in the incidence of breast cancer in postmenopausal women (25). The present data demonstrated that iron overload induced by erastin exhibited a good antitumor effect via inducing autophagic death in breast cancer cells. Breast cancer develops in women as a result of multiple factors, which include diet, socioeconomic status and genetic mutations (25). Therefore, the relationship between iron and breast cancer requires further investigation. Ferroptosis is a novel type of regulated cell death that is accompanied by intracellular iron accumulation (14). Activating ferroptosis to eliminate breast cancer cells has emerged as a potential therapeutic approach. Erastin is an inducer of ferroptosis that has been reported to efficiently induce death in various types of cancer cells, such as liver cancer (10) and rhabdomyosarcoma cells (12). Consistently, the present study demonstrated that erastin inhibited the viability of breast cancer cells and induced cell death in MCF-7 and MDA-MB-231 cells in a dose-dependent manner.

A previous study has reported that erastin induces ferroptosis in triple-negative MDA-MB-231 breast cancer cells (13). The current study demonstrated that erastin activated autophagy in triple-negative MDA-MB-231 and estrogen receptor-positive MCF-7 breast cancer cell lines. Beclin1, ATG5, ATG12, LC3B and p62 are key autophagic marker proteins whose levels reflect the occurrence of autophagy (8). In the present study, western blotting revealed that protein expression levels of Beclin1, ATG5, ATG12 and LC3B were increased, while p62 levels were decreased in a time-dependent manner following erastin treatment. Autophagy-inhibition using 3-MA, Baf-A1 and ATG5-knockdown via siRNA inhibited the erastin-induced effects on autophagy-related proteins and cell death.

Ferroptosis is a type of programmed cell death induced by iron-dependent lipid peroxidation (26). Iron, as a promoter of cell growth and proliferation, fulfils an important role in human diseases, such as Parkinson's (27). An abnormal increase of intracellular iron in the absence of erastin has also been reported to induce cell death (3). Fang et al (28) used ferric ammonium citrate (FAC) and a membrane-permeable ferric 8-hydroxyquinoline complex (Fe-8HQ), which are two types of iron agents, to improve intracellular iron levels. It was identified that either FAC or Fe-8HQ induced death in various types of cells in a dose-dependent manner. Another study reported by Nakamura et al (29) demonstrated that iron overload could improve intracellular ROS levels, which then contribute to cell death via activating the MAPK signaling pathway. The Fenton reaction, which recycles $\mathrm{Fe}^{2+}$, is a key step in ferroptosis that produces ROS, contributing to cell death (15). Therefore, improving iron levels may suppress tumor growth and enhance the anticancer activity of ferroptosis inducers (27). However, the mechanism of erastin-induced cell death in breast carcinoma remains unclear. In the present study, the iron assay revealed that intracellular iron accumulates in breast cancer cells following treatment with erastin.

Erastin induces breast cancer cell death and increases intracellular iron levels (6). However, to the best of our knowledge, the relationship between erastin-induced autophagic cell death and iron levels in breast carcinoma is unknown. Previously, autophagy has been demonstrated to contribute to ferroptosis via increasing intracellular iron level by degradation of nuclear receptor coactivator 4 , which is an endogenous inhibitor of ferritin (7). However, it remains unknown whether iron plays a role in autophagy activation. Thus, the present study used breast cancer cells and investigated the role of iron in erastin-induced lethal autophagy. The current study therefore pre-treated cells with an iron chelator (DFO) prior to erastin treatment. As a result, the accumulation of intracellular iron was inhibited and cell death was decreased. The results further revealed that DFO inhibited the expression of autophagy-related proteins affected by erastin. The results demonstrated that the mitigation of erastin-induced irons levels inhibited erastin-induced autophagy. The current results indicated that abnormal improvement of intracellular iron could activate autophagic cell death in breast cancer cells.

Despite not elucidating the precise mechanism by which iron levels inhibit erastin-induced autophagy, previous studies have provided results supporting those of the current study. Numerous studies have demonstrated that iron excess and oxidative stress may lead to ROS accumulation, which damages cells and activates autophagy $(21,30,31)$. Excessive autophagy contributes to ferroptosis by regulating cellular iron equilibria (30). A previous study reported that erastin induces autophagy by depleting GSH, which increases lipid ROS generation $(32,33)$. Therefore, iron improvement could activate autophagy via ROS-related pathways, such as AMPK/mTOR (29). Mitochondria are regarded as the location where ROS, as a crucial messenger, are produced via electron transmission chain (34). Furthermore, some pro-death factors, such as cytochrome $\mathrm{C}$, nuclease endo $\mathrm{G}$ and apoptosis inducing factor, are also located within mitochondria, and can induce cell death after being released when mitochondria are impaired (34). Although mitochondria are also the location of energy generation; glycolysis is the primary pathway of ATP generation in cancer cells (34). However, whether the autophagy activated by erastin could inhibit the function of mitochondria remains to be investigated further. In the present study, DFO inhibited erastin-induced autophagy and improved intracellular iron levels. Thus, it was concluded that ROS serves an important role in the relationship between iron level improvement and erastin-induced autophagy.

In conclusion and to the best of our knowledge, the current study demonstrated for the first time that erastin triggers autophagy in breast cancer cells by improving intracellular iron levels.

\section{Acknowledgements}

Not applicable.

\section{Funding}

No funding was received.

\section{Availability of data and materials}

The datasets used and/or analyzed during the present study are available from the corresponding author on reasonable request. 


\section{Authors' contributions}

MXL, PFG and DS designed the research and analyzed the data. MXL, XZW and SL performed the experiments. $\mathrm{CH}$, $\mathrm{CCW}, \mathrm{LW}$ and XYW were responsible for acquisition of data, analysis and interpretation of data. All authors read and approved the final manuscript.

\section{Ethics approval and consent to participate}

Not applicable.

\section{Patient consent for publication}

Not applicable.

\section{Competing interests}

The authors declare that they have no competing interests.

\section{References}

1. Choi J, Gyamfi J, Jang H and Koo JS: The role of tumor-associated macrophage in breast cancer biology. Histol Histopathol 33: 133-145, 2018

2. Fisusi FA and Akala EO: Drug combinations in breast cancer therapy. Pharm Nanotechnol 7: 3-23, 2019.

3. Nedeljković M and Damjanović A: Mechanisms of chemotherapy resistance in triple-negative breast cancer-how we can rise to the challenge. Cells 8: 957, 2019.

4. Maldonado EN, Sheldon KL, DeHart DN, Patnaik J, Manevich Y, Townsend DM, Bezrukov SM, Rostovtseva TK and Lemasters JJ: Voltage-Dependent anion channels modulate mitochondrial metabolism in cancer cells: Regulation by free tubulin and erastin. J Biol Chem 288: 11920-11929, 2013.

5. Xu T, Ding W, Ji X, Ao X, Liu Y, Yu W and Wang J: Molecular mechanisms of ferroptosis and its role in cancer therapy. J Cell Mol Med 23: 4900-4912, 2019.

6. Dixon SJ, Lemberg KN, Lamprecht MR, Skouta R, Zaitsev EM, Gleason CE, Patel DN, Bauer AJ, Cantley AM, Yang WS, et al: Ferroptosis: An iron-dependent form of nonapoptotic cell death. Cell 149: 1060-1072, 2012

7. Gao M, Monian P, Pan Q, Zhang W, Xiang J and Jiang X: Ferroptosis is an autophagic cell death process. Cell Res 26 : 1021-1032, 2016

8. Zhou B, Liu J, Kang R, Klionsky DJ, Kroemer G and Tang D: Ferroptosis is a type of autophagy-dependent cell death. Semin Cancer Biol 14 (Suppl): S1044-S1579, 2019.

9. Kang R and Tang D: Autophagy and ferroptosis-What's the connection? Curr Pathobiol Rep 5: 153-159, 2017.

10. Chen Y, Zhu G, Liu Y, Wu Q, Zhang X, Bian Z, Zhang Y, Pan Q and Sun F: O-GlcNAcylated c-jun antagonizes ferroptosis via inhibiting GSH synthesis in liver cancer. Cell Signal 63: 109384 , 2019.

11. Dächert J, Schoeneberger H, Rohde K and Fulda S: RSL3 and erastin differentially regulate redox signaling to promote smac mimetic-induced cell death. Oncotarget 7: 63779-63792, 2016.

12. Dächert J, Ehrenfeld V, Habermann K, Dolgikh N and Fulda S: Targeting ferroptosis in rhabdomyosarcoma cells. Int J Cancer 15: 510-520, 2019

13. Yu M, Gai C, Li Z, Ding D, Zheng J, Zhang W, Lv S and Li W: Targeted exosomes-encapsulated erastin induced ferroptosis in the triple negative breast cancer cells. Cancer Sci 110: 3173-3182, 2019.

14. Vanden Berghe T, Linkermann A, Jouan-Lanhouet S, Walczak H and Vandenabeele P: Regulated necrosis: The expanding network of non-apoptotic cell death pathways. Nat Rev Mol Cell Biol 15: 135-147, 2014.
15. Doll S and Conrad M: Iron and ferroptosis: A still ill-defined liaison. IUBMB Life 69: 423-434, 2017.

16. Kagan VE, Mao G, Qu F, Angeli JP, Doll S, Croix CS, Dar HH, Liu B, Tyurin VA, Ritov VB, et al: Oxidized arachidonic and adrenic PEs navigate cells to ferroptosis. Nat Chem Biol 13: 81-90, 2017.

17. Hou W, Xie Y, Song X, Sun X, Lotze MT, Zeh JZ III, Kang R and Tang D: Autophagy promotes ferroptosis by degradation of ferritin. Autophagy 12: 1425-1428, 2016.

18. Schroeder B, Schulze RJ, Weller SG, Sletten AC, Casey CA and McNiven MA: The small GTPase Rab7 as a central regulator of hepatocellular lipophagy. Hepatology 61: 1896-1907, 2015.

19. Song X, Zhu S, Chen P, Hou W, Wen Q, Liu J, Xie Y, Liu J, Klionsky DJ, Kroemer G, et al: AMPK-Mediated BECN1 phosphorylation promotes ferroptosis by directly blocking system Xc(-) activity. Curr Biol 28: 2388-2399, 2018.

20. Brown CW, Amante JJ, Goel HL and Mercurio AM: The $\alpha 6 \beta 4$ integrin promotes resistance to ferroptosis. J Cell Biol 216 4287-4297, 2017.

21. Wu Z, Geng Y, Lu X, Shi Y, Wu G, Zhang M, Shan B, Pan H and Yuan J: Chaperone-Mediated autophagy is involved in the execution of ferroptosis. Proc Natl Acad Sci USA 116: 2996-3005, 2019.

22. Zhou Z, Lu B, Wang C, Wang Z, Luo T, Piao M, Meng F, Chi G, Luo Y and Ge P: RIP1 and RIP3 contribute to shikonin-induced DNA double-strand breaks in glioma cells via increase of intracellular reactive oxygen species. Cancer Lett 390: 77-90, 2017.

23. Pan H, Wang Y, Na K, Wang Y, Wang L, Li Z, Guo C, Guo D and Wang X: Autophagic flux disruption contributes to ganoderma lucidum polysaccharide-induced apoptosis in human colorectal cancer cells via MAPK/ERK activation. Cell Death Dis 10: 456, 2019.

24. Choi J, Jo M, Lee E, Oh YK and Choi D: The role of autophagy in human endometrium. Biol Reprod 86: 70, 2012.

25. Huang X: Does iron have a role in breast cancer? Lancet Oncol 9: 803-807, 2008.

26. Wang Z, Ding Y, Wang X, Lu S, Wang C, He C, Wang L, Piao M, Chi G, Luo Y and Ge P: Pseudolaric acid B triggers ferroptosis in glioma cells via activation of Nox4 and inhibition of Xct. Cancer Lett 428: 21-33, 2018.

27. Wang S, Luo J, Zhang Z, Dong D, Shen Y, Fang Y, Hu L, Liu M, Dai C, Peng S, et al: Iron and magnetic: New research direction of the ferroptosis-based cancer therapy. Am J Cancer Res 8: 1933-1946, 2018.

28. Fang S, Yu X, Ding H, Han J and Feng J: Effects of intracellular iron overload on cell death and identification of potent cell death inhibitors. Biochem Biophys Res Commun 503: 297-303, 2018.

29. Nakamura T, Naguro I and Ichijo H: Iron homeostasis and iron-regulated ROS in cell death, senescence and human diseases. Biochim Biophys Acta Gen Subj 1863: 1398-1409, 2019.

30. Torii S, Shintoku R, Kubota C, Yaegashi M, Torii R, Sasaki M, Suzuki T, Mori M, Yoshimoto Y, Takeuchi T and Yamada K: An essential role for functional lysosomes in ferroptosis of cancer cells. Biochem J 473: 769-777, 2016.

31. Byun YJ, Kim SK, Kim YM, Chae GT, Jeong SW and Lee SB: Hydrogen peroxide induces autophagic cell death in C6 glioma cells via BNIP3-mediated suppression of the mTOR pathway. Neurosci Lett 18: 131-135, 2009.

32. Sun Y, Zheng Y, Wang C and Liu Y: Glutathione depletion induces ferroptosis, autophagy, and premature cell senescence in retinal pigment epithelial cells. Cell Death Dis 9: 753, 2018.

33. Mancilla H, Maldonado R, Cereceda K, Villarroel-Espindola F, Montes de Oca M, Angulo C, Castro MA, Slebe JC, Vera JC, Lavandero S and Concha II: Glutathione depletion induces spermatogonial cell autophagy. J Cell Biochem 116: 2283-2292, 2015.

34. Yang Y, Karakhanova S, Hartwig W, D'Haese JG, Philippov PP, Werner J and Bazhin AV: Mitochondria and mitochondrial ROS in cancer: Novel targets for anticancer therapy. J Cell Physiol 231: 2570-2581, 2016

This work is licensed under a Creative Commons Attribution-NonCommercial-NoDerivatives 4.0 International (CC BY-NC-ND 4.0) License. 